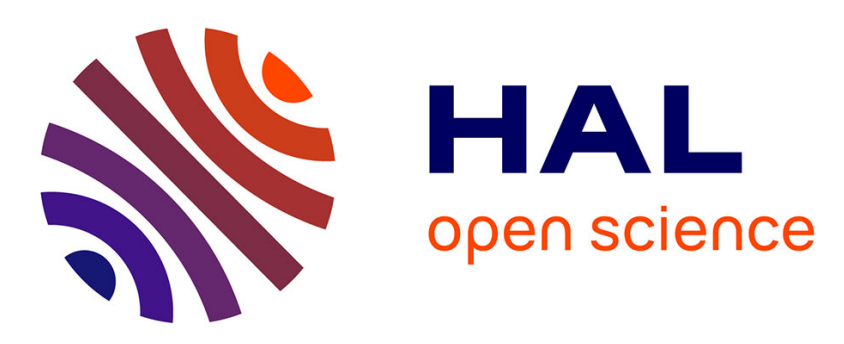

\title{
Photoreceptor detection in in-vivo Adaptive Optics images of the retina: Towards a simple interactive tool for the physicians
}

Kevin Loquin, Isabelle Bloch, Kiyoko Nakashima, Florence Rossant, Michel Paques

\section{To cite this version:}

Kevin Loquin, Isabelle Bloch, Kiyoko Nakashima, Florence Rossant, Michel Paques. Photoreceptor detection in in-vivo Adaptive Optics images of the retina: Towards a simple interactive tool for the physicians. ISBI: International Symposium on Biomedical Imaging, Mar 2011, Chicago, IL, United States. 10.1109/ISBI.2011.5872385 . hal-01959833

\section{HAL Id: hal-01959833 https://hal.science/hal-01959833}

Submitted on 19 Dec 2018

HAL is a multi-disciplinary open access archive for the deposit and dissemination of scientific research documents, whether they are published or not. The documents may come from teaching and research institutions in France or abroad, or from public or private research centers.
L'archive ouverte pluridisciplinaire HAL, est destinée au dépôt et à la diffusion de documents scientifiques de niveau recherche, publiés ou non, émanant des établissements d'enseignement et de recherche français ou étrangers, des laboratoires publics ou privés. 


\title{
PHOTORECEPTOR DETECTION IN IN-VIVO ADAPTIVE OPTICS IMAGES OF THE RETINA: TOWARDS A SIMPLE INTERACTIVE TOOL FOR THE PHYSICIANS
}

\author{
Kevin Loquin $^{\star *} \quad$ Isabelle Bloch ${ }^{\star} \quad$ Kiyoko Nakashima Florence Rossant $^{\ddagger} \quad$ Michel Paques $^{\dagger}$ \\ * Institut Telecom - Telecom ParisTech - CNRS LTCI - Paris, France \\ Kevin.Loquin@telecom-paristech.fr Isabelle.Bloch@telecom-paristech.fr \\ ${ }^{\dagger}$ Clinical Investigation Center 503 of the Quinze-Vingts Hospital DHOS/INSERM - Paris, France \\ knecicoph.org mpecicoph.org \\ ${ }^{\ddagger}$ ISEP - Paris, France \\ Florence.Rossant@isep.fr
}

\begin{abstract}
This article presents a novel photoreceptor detection algorithm applied to in-vivo Adaptive Optics (AO) images of the retina obtained from an advanced ophthalmic diagnosis device. Our algorithm is based on a recursive construction of thresholded connected components when the seeds of the recursions are the regional maxima of the image. This algorithm results in a labeling of the AO image which is then used to segment the image with a marker-controlled watershed algorithm. This method has been implemented in a software currently used by medical experts, and preliminary results are very encouraging.
\end{abstract}

Index Terms - Adaptive Optics, Photoreceptor detection, in vivo diagnosis, retina imaging

\section{INTRODUCTION}

The light-sensitive tissue of the eye is the retina, which covers the eye fundus. At a microscopic level, the retina is a stack of several neuronal layers whose aim is to transform the incident light in an electrical signal (phototransduction) that will be transmitted to the brain (Figure 1) [1,2]. The photosensitive neurons are the photoreceptor cells (although some ganglion cells are responsive to light). These are of two types: rods and cones. Rods function mainly in dim light and provide black-and-white vision, while cones support daytime vision and the perception of colour. Many inherited and acquired diseases or disorders may affect the retina [3, 4]. Some of these provoke progressive degeneration of the retina. While the loss of rods is usually well tolerated in everyday life, loss of cones leads to severe visual handicap. In some diseases such as retinitis pigmentosa, visual loss can occur very early in life. Yet, in most cases, visual acuity usually deteriorates gradually over decades and may end up to mere light perception.

In recent years, it became possible to image the living human retina at the microscopic scale by means of new imaging systems: Adaptive Optics (AO) [5, 6]. The AO images presented in this article are obtained with a prototypic AO system (rtxl Adaptive Optics Retinal Camera manufactured by Imagine Eyes, Orsay, France), which is currently in operation in a clinical setting at the Clinical Investigation Center 503 of the $X V-X X$ hospital. Each acquisition is a sequence of forty $1392 \times 1040$ en-face images. In each image,

\footnotetext{
${ }^{*}$ This work has been supported by French National Research Agency (ANR) through Tecsan Program (project iPhot $n^{o}$ ANR-09-TECS-009).
}

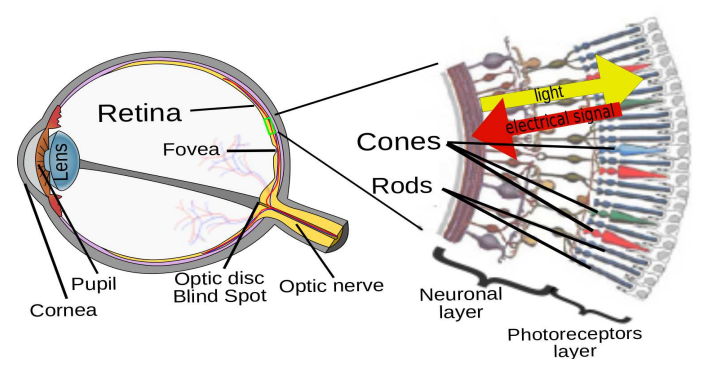

Fig. 1. Schematic eye and retina. This figure is based on materials obtained from http://www.mrawde.com/blog/2008/05/readingin-the-dark.html and http://webvision.med.utah.edu/sretina.html.

considering a mean axial length of $24.7 \mathrm{~mm}$, one pixel corresponds to a $1.6 \mu \mathrm{m} \times 1.6 \mu \mathrm{m}$ spot on the retina. The images that we are dealing with are shown in Figure 2. They are the result of a registration/summation algorithm applied to the forty images acquired successively, in order to increase the signal-to-noise ratio.

The obtained AO images are very useful to a physician. This remark is illustrated in Figure 2, where a non degenerative (image a) and degenerative cases (image b, c, d) can easily be differentiated. In [7], the authors illustrate the diagnosis power of retinal AO images on degenerative cones distrophy by comparing manual diagnosis procedures from these $\mathrm{AO}$ images with other usual diagnosis procedures.

Automated retinal AO image analysis methods for assisting in vivo diagnosis and follow up of early degenerative cones dystrophy are essential for physicians. In this paper, we propose a novel algorithm that aims at detecting the photoreceptors in the AO images (Figure 2). The cornerstone of all the AO images based diagnosis procedures presented in [7] is the detection of the cones in these images. From these detected cones, we can derive photoreceptor density maps or extract other statistical features useful for the diagnosis.

Automatic photoreceptors detection algorithms have already been proposed in the literature. In [8], the authors proposed a multistep algorithm. First, they propose to work with an above-threshold version of the image in order to eliminate the dim spots. Afterwards, the regional maxima are detected from a low-pass filtered version of the thresholded image in order to eliminate the noise. Finally, a morphological dilation is used to merge close enough detected spots. The structuring element is chosen according to the minimal 

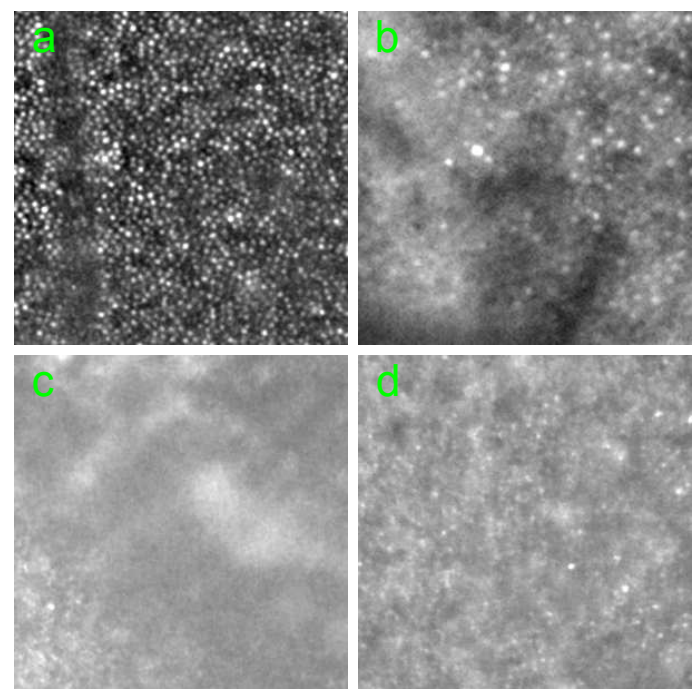

Fig. 2. AO images of a: a healthy eye. b: Retinitis Pigmentosa (RP) c: Age-related Macular Distrophy (AMD) due to Drusen. d: Occult Macular Distrophy (OMD).

cone spacing. In [9], the same kind of procedure is proposed but the regional maxima detection is performed by decreasing greylevel regions in order to favor the brightest detected spots and avoid the threshold step of the method in [8].

We distance these articles by providing a new low-level image processing algorithm which is computationally very efficient. The geometrical arguments from which we construct our algorithm are the same as the ones used in $[8,9]$ : elimination of dim spots and merging of too close spots. Our algorithm is simpler and more computationally efficient because directly constructed from the image geometry and these geometric arguments. It is based on a recursive construction of thresholded connected components when the seeds of the recursions are the regional maxima of the image. The stopping rules of this recursive construction are deduced from the previous geometrical arguments and the finally detected photoreceptors are the highest greylevel spots in a merged maximum area (a thresholded connected component).

The efficiency of our algorithm and its simplicity allows a visual and adaptive control of the algorithm parameterization, which makes it easily tunable by non computer scientists like physicians. An important facet of our work stands in the usability of our tool, implemented as an ImageJ plugin in collaboration with physicians.

In Section 2, we present and geometrically justify our photoreceptor detection algorithm. Then, Section 3 details the main user interactions provided with our implemented tool. Finally, in Section 4 , we illustrate the results of our method when applied to various images and propose a simple visual comparison with the method proposed in [8], before concluding.

\section{PHOTORECEPTOR DETECTION ALGORITHM}

In order to illustrate the algorithm functioning, we accompany the following discussion about the algorithm with Figure 3 which shows the algorithm result for five different tolerance parameter (explained below) choices on a unique 1-dimensional grey level signal and with Figure 4 which shows the result of our labeling algorithm when applied to a medical AO image of the retina.
In the used AO images, the photoreceptors that we aim at detecting are bright spots, i.e. pixels with high grey levels. Hence a first natural step of our algorithm is a simple regional maxima detection. A regional maximum is a plateau of the grey level function, i.e. a connected component of a level $\alpha$ in which every pixel has a grey level equal to $\alpha$.This terminology is reused from existing works on the component trees found in the literature [10]. These regional maxima appear as RM on the top-left diagram of Figure 3.
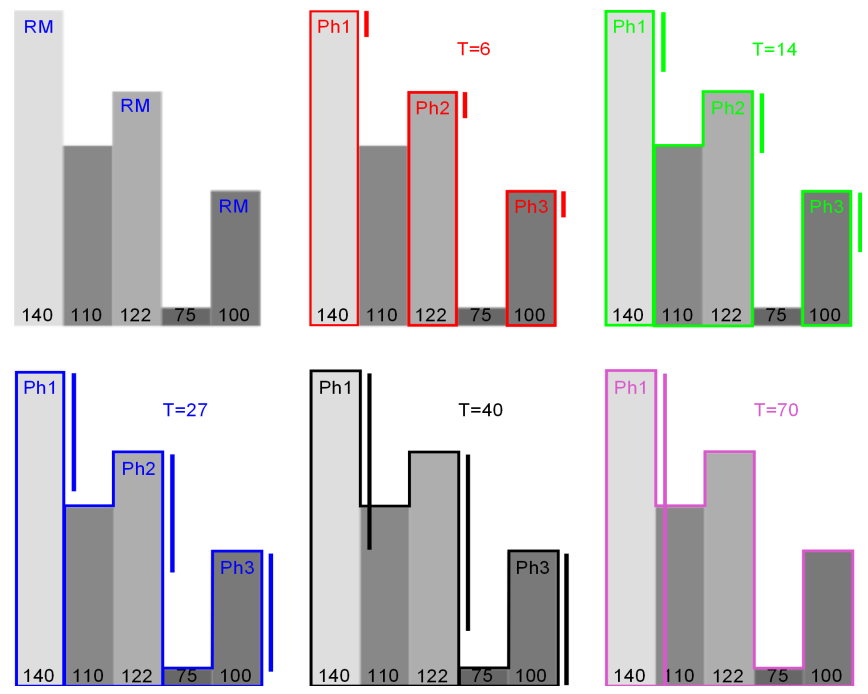

Fig. 3. Illustration of the algorithm.

This raw regional maxima processing is not enough to qualify these regional maxima as the photoreceptors that we aim at detecting. Indeed, a regional maximum with a low greylevel (i.e. a dim spot) is not generally a photoreceptor. The thresholding of the AO image is the solution proposed in [8] to eliminate these spots. Besides, too close regional maxima can be due to a unique photoreceptor. This idea led the authors of $[8,9]$ to morphologically filter the extracted regional maxima. In our approach, we define a geometrical concept of influence area of a maximum that we will call a maximum area. The construction of these maximum areas induces the elimination of the undesired spots. These maximum areas appear as colored rectangles in Figure 3 for different tolerances.

A maximum area of a regional maximum is defined up to a tolerance parameter, denoted by $T$. This tolerance parameter finds its roots in the component tree decomposition of an image [10]. In this fructuous research field, an image is seen as a decomposition of $\alpha$ level connected components. The level range corresponding to the greyscale range of the image, an image is decomposed in a stack of connected components. The tolerance parameter $T$ that we are using in our algorithm tunes the height of the top part of this stack that we consider as a maximum area. The top parts of the stacks appear as colored bars in Figure 3. When the color bar (or the tolerance top level) intersects a pixel, this pixel is incorporated in the maximum area of the associated regional maximum.

It is trivial to remark that the higher the tolerance value, the bigger the maximum areas of the regional maxima. Going further, for a given $T$, two or more regional maxima can lead to the same maximum area. In Figure 3, when the tolerance is $T=40$ we can observe the fusion of $\mathrm{Ph} 1$ and $\mathrm{Ph} 2$ into one photoreceptor: $\mathrm{Ph} 1$ which is of higher grey level. This is the first criterion that we use to eliminate regional maxima of smallest grey levels, seeding the same fused 


\section{maximum area.}

More technically, our algorithm constructs the connected component rooted by all the regional maxima, taken in a decreasing greylevel order. For each regional maximum, this construction is recursive around the considered regional maximum and the recursivity is stopped if we reach a pixel

- whose greylevel is out of the top stack of the image, i.e. out of the connected component defined from the considered regional maximum by means of the tolerance; or

- already processed from another regional maximum: it means that we reach a connected component of higher greylevel regional maximum (the fusion of too close spots is performed here by keeping only the brightest spot in a connected component. Besides a filtering of the dimer spots is implicitely performed); or

- whose greylevel is higher than the greylevel of the initial regional maximum: it occurs when the reached higher greylevel pixel was not itself a regional maximum because one of its direct neighbors is higher (Once again, an implicit filtering of the dim spots is performed here).

More than filtering the roughly detected regional maxima, the implemented algorithm labels the pixels since it is followed by the application of a marker-controlled watershed segmentation algorithm. Therefore, at each pixel is associated one of the following labels:

- $M A X_{-} P O I N T$ : a finally detected photoreceptor,

- EQUAL: inside a regional maximum,

- $M A X \_A R E A$ : inside a maximal area.

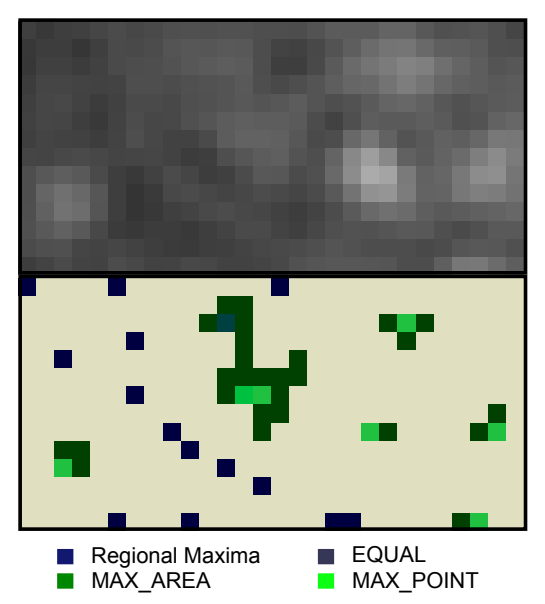

Fig. 4. Pixel labeling illustration.

Note that the proposed algorithm is a truncated adaptation of the watershed by immersion algorithm [11] when applied to the inverted image. In the inverted image, the origins of the basins used in the watershed by immersion are the regional maxima of the original image. In our case, we are not interested in reaching a complete separation of the basins (the maxima) but only in elicitating these basins until a given height, i.e. the maximum areas up to a tolerance parameter).

\section{ALGORITHM IMPLEMENTATION: AN INTERACTIVE TOOL FOR THE PHYSICIANS}

This algorithm is very rapid: after a complete cover of the image for finding regional maxima, the recursive coverage is only applied to the extracted regional maxima which highly diminishes the computational cost. When java-implemented as an imageJ plugin (which is not the most rapid computational framework) the algorithm result is instantly displayable. This remark is very important because it allows us to provide an interactive tool to the final user, the physician. This non algorithmic part of our work is greatly appreciated by the physicians who can thus easily interact with the algorithm and adapt its parameters to his visual expertise in an intuitive way. This feature highly contributes to the fact that, even if still in development, this tool is already in use in a clinical context.

Among the proposed interactions, the parameterization (more precisely the tolerance value choice) is the most important. The highest the tolerance value, the smallest the number of detected cones. As illustrated in Figure 2, the images are highly different according to the involved disease or the acquisition conditions. It does not seem possible to find an automatic robust optimal tolerance selection method, but it is still one of our research interests. Thus, in the tool that we develop and implement in collaboration with the physicians, we proposed the solution of incorporating a scrollbar to tune the tolerance parameter. Each time the user moves this scrollbar, the detected photoreceptors directly appear on a refreshed image. Thus, the physician is the judge of the best trade-off between wrong detected cones and undetected cones.

Another way to interact on the photoreceptors detection is to incorporate in our image J plugin the possibility to add non automatically detected cones and to remove wrongly detected cones by the hand. Note that in a following work, we propose to perform a statistical study of these two indices: the number of added cones and the number of removed cones in order to evaluate our detection algorithm. We assume that these indices quantitatively reflect the subjective judgement of the physician about our algorithm.

\section{ILLUSTRATIVE EXPERIMENTS}

In order to illustrate the results of our algorithm on AO images of the retina, we work with the images of Figure 2. All of them have the same size: $300 \times 300$ (i.e. $480 \mu m \times 480 \mu m$ ). The first column in Figure 5 presents the photoreceptors automatically detected with our algorithm for respective tolerance of $T=20, T=30, T=30$ and $T=34$. The tolerance has been interactively chosen by the authors with an aim of visualizing the results at best. No manual removal or addition of photoreceptors have been made here. The second column presents the results obtained with the algorithm of [8]. The parameters are $f_{c}$ : the cutoff frequency of the used lowpass filter and $t h r$ is the chosen threshold. For proper parameterizations, the obtained results are very similar for these methods. Nevertheless, with our tool, the physicians have a less esoteric parameterization procedure that leads to more usability.

Figure 6 presents the results that we obtain with the markercontrolled watershed segmentation results using the same tolerance parameters as in the previous experiment. The segmentation result is very useful to physicians. First it exhibits parts of low cone density when the segmented parts are big (see Figure $6 \mathbf{b}, \mathbf{c}$ and $\mathbf{d}$ ). In other words, the segmentation is a representation of information dual to a cone density map. Besides, the segmentation result provides information about the cone mosaic configuration (e.g. hexagonal) in the high cone density parts (see Figure 6 a). The extracted quantitative topographical information is of primary importance especially in the context of longitudinal follow-ups of cone degenerative distrophies. This is a real advantage for the physician to accompany his visual judgment with a quantization of the disease evolution. 

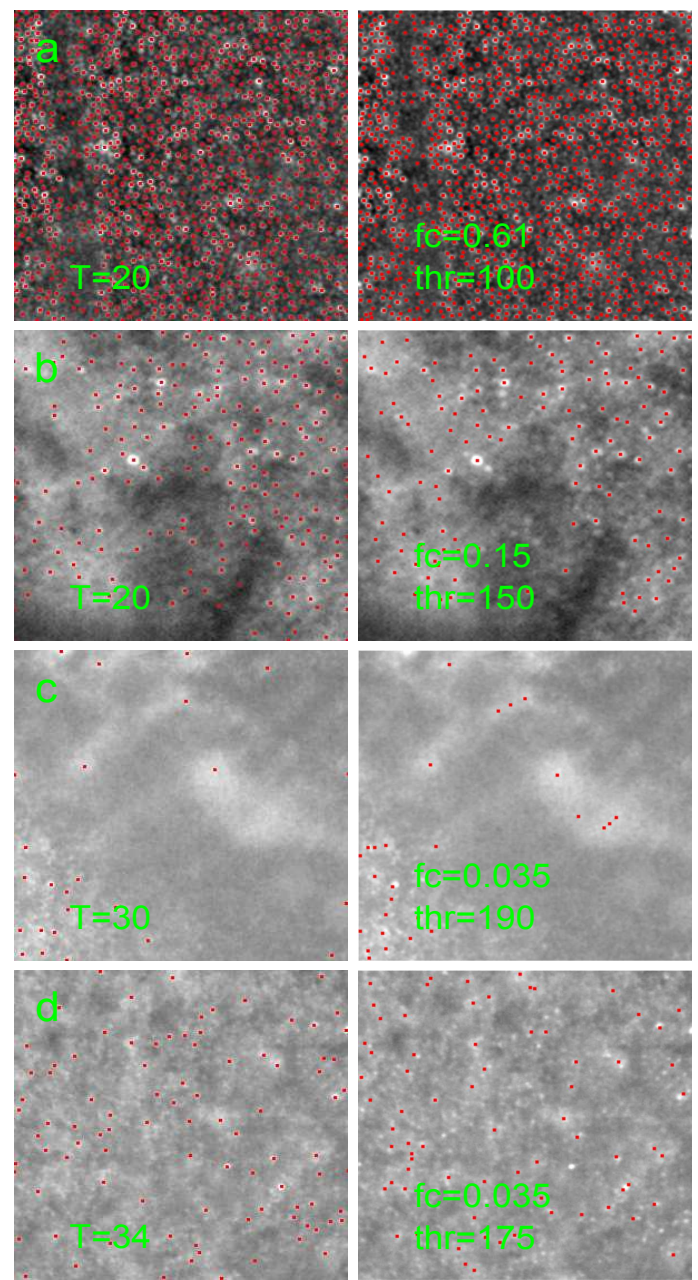

Fig. 5. Comparative experiment: our algorithm (left column) vs. Li and Roorda algorithm [8] (right column) results when applied to the AO images of Figure 2.

\section{CONCLUSION}

In this paper, we proposed a new photoreceptor detection algorithm applied to in-vivo AO images of the retina. This algorithm is based on a recursive construction of thresholded connected components when the seeds of the recursions are the regional maxima of the image. Our method is implemented as an imageJ plugin already in use in a clinical setting at the $X V-X X$ hospital. This use shows its early clinical value before any objective evaluation. In a raw comparative study, our algorithm seems to provide at least as good results (in terms of detection efficiency) as already existing approaches. The main advantage of our approach stands more in its computational efficiency, which is highly appreciated by the physicians especially because we provide an interactive parameterization, allowing an optimal (according to the physician expertise) detection. Note that a semi-subjective evaluation protocol is currently in progress with the $X V-X X$ hospital: a statistical study of correction indices derived from the number of manually removed and added cones is established.

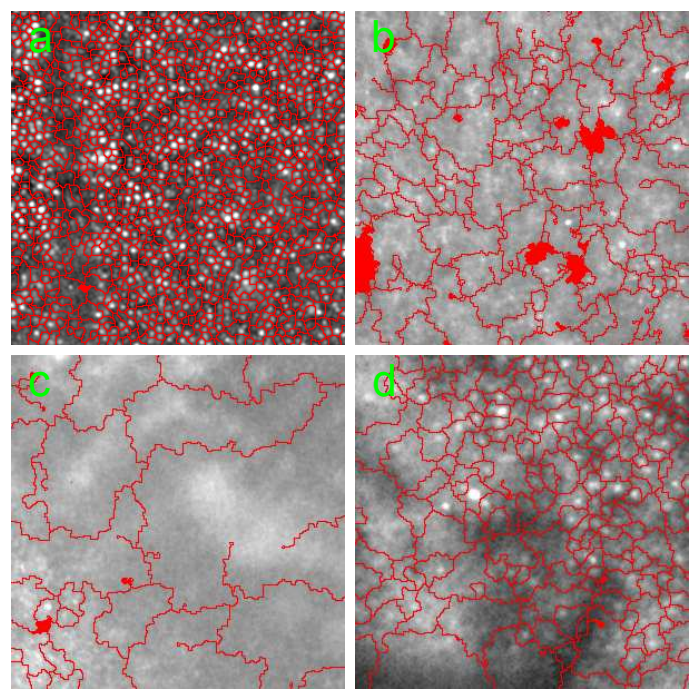

Fig. 6. Marker-controlled watershed segmentation results when applied to the AO images of Figure 2.

\section{REFERENCES}

[1] S.L. Polyak, The Retina, University of Chicago Press, 1941.

[2] C.A. Curcio, K.R. Sloan KR, R.E. Kalina, and A.E. Hendrickson, "Human photoreceptor topography," The Journal of comparative neurology, vol. 292(4), pp. 497-523, 1990.

[3] S.J. Ryan, D.R. Hinton, A.P. Schachat, and P. Wilkinson, Retina (3 Volume Set), Mosby, 2005.

[4] P.T. de Jong, "Age-related macular degeneration," The New England Journal of Medicine, vol. 355(14), pp. 1474-1485, 2006.

[5] F. Roddier, Adaptive Optics in Astronomy, Cambridge University Press, 1999.

[6] A. Roorda, F. Romero-Borja, W. Donnelly, H. Queener, T. Hebert, and M. Campbell, "Adaptive optics scanning laser ophthalmoscopy," Optics Express, vol. 10(9), pp. 405-412, 2002.

[7] J. I. Wolfing, M. Chung, J. Carroll, A. Roorda, and D. R. Williams, "High-resolution retinal imaging of cone-rod dystrophy," Ophthalmology, vol. 113, pp. 1014-1019, 2006.

[8] K.Y. Li and A. Roorda, "Automated identification of cone photoreceptors in adaptive optics retinal images," Journal of the Optical Society of America A, vol. 24(5), pp. 1358-1363, 2007.

[9] B. Xue, S.S. Choi, N. Doble, and J.S. Werner, "Photoreceptor counting and montaging of enface retinal images from an adaptive optics fundus camera," Journal of the Optical Society of America A, vol. 24(5), pp. 1364-1372, 2007.

[10] M.Couprie, L. Najman, and G. Bertrand, "Quasi-linear algorithms for the topological watershed," Journal of Mathematical Imaging and Vision, vol. 22, no. 2-3, pp. 231-249, 2005.

[11] L. Vincent and P. Soille, "Watersheds in digital spaces: an efficient algorithm based on immersion simulations," IEEE Trans. Patt. Anal. Mach. Intell., vol. 13, no. 6, pp. 583-598, 1991. 